\title{
Case Report: Coffin-Lowry Syndrome: The First Molecularly Confirmed Report in Iran
}

\author{
Ali Nikfar ${ }^{1,2}$ (D), Mojdeh Mansouri' ${ }^{1}$ (D) Gita Fatemi Abhari²
}

1. Department of Genetics and Molecular Medicine, School of Medicine, Zanjan University of Medical Sciences, Zanjan, Iran

2. Imam Khomeini Genetic Counseling Center, Welfare Organization of Zanjan, Zanjan, Iran.

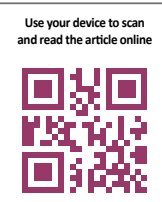

citation: Nikfar A, Mansouri M, Fatemi Abhari G. Coffin-Lowry Syndrome: The First Molecularly Confirmed Report in Iran. Iranian Rehabilitation Journal. 2018; 16(3):325-329. http://dx.doi.org/10.32598/irj.16.3.325

http://dx.doi.org/10.32598/irj.16.3.325

\section{(i) (3)}

Funding: See Page 328

Article info:

Received: 29 Mar 2018

Accepted: 20 Jul 2018

Available Online: 01 Sep 2018

Keywords:

Coffin-lowry Syndrome, RPS6KA3, RSK2, X-linked mental retardation, Whole exome sequencing

\section{ABSTRACT}

Coffin-Lowry syndrome (CLS) is an X-linked disorder, which affects hemizygous males more severely than females. It is characterized by mental retardation, short stature, head and facial abnormalities, skeletal anomalies and developmental delays. The signs and symptoms vary in different people. We report a 14-year-old male patient, diagnosed with CLS based on his clinical features. Genetic testing revealed a de novo mutation in ribosomal protein S6 kinase alpha-3 (RPS6KA3) gene (c.2185C $>\mathrm{T} ; \mathrm{p}$. Arg729Trp). This is the first molecularly confirmed case report of a patient with CLS from Iran.

\section{Highlights .}

- Coffin-Lowry Syndrome is caused by mutations in the ribosomal protein S6 kinase alpha-3 (RPS6KA3) gene, which encodes ribosomal S6 kinase-2 (RSK2), a growth factor-regulated protein kinase.

- In the current study, Whole Exome Sequencing (WES) revealed a de novo missense mutation (c.2185C $>\mathrm{T}$; p.Arg729Trp) in exon 22 of RPS6KA3 gene.

- Whole Exome Sequencing (WES) seems to be an efficient and sensitive method to identify possible diseasecausing mutations.

- To the best of our knowledge, this is the first molecularly confirmed report of CLS in Iran. 


\section{Plain Language Summary}

Coffin-Lowry Syndrome (CLS) is a rare genetic disorder with X-linked dominant inheritance affecting multiple systems. Approximately $80 \%$ of patients have no history of this illness in their families. Signs and symptoms may include intellectual disability, delayed development, short stature, skeletal abnormalities and characteristic dysmorphism, most notably affecting the face and hands. Mutations in the RPS6KA3 gene cause this syndrome. This gene provides instructions for synthesis of a protein involved in signaling within cells. Treatment is symptomatic and supportive. Prognosis is poor and depends on the severity of the disease. Early intervention may improve the outlook for patients. Diagnosis is usually based on the clinical presentation. Early on, RPS6KA3 mutation analysis may be used for rapid diagnosis. Here, we elaborate the clinical and genetic analysis of an Iranian patient with CLS. He was the second child of nonconsanguineous parents. Clinical examinations revealed signs and symptoms of CLS. His karyotype was normal. We applied Whole Exome Sequencing (WES), a genomic technique for sequencing all of the protein-coding genes in a genome, to identify the causal variant in the patient. Results from WES revealed a hemizygous missense variant in exo 22 (c.2185C $>$ T; p.Arg729Trp) of RPS6KA3 gene. To the best of our knowledge, this is the first report of molecularly confirmed case of CLS in the Iranian population.

\section{Introduction}

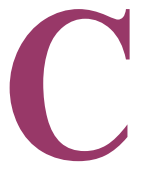

offin-Lowry syndrome (CLS, OMIM 303600 ) is a rare $\mathrm{X}$-linked intellectual disability syndrome characterized by mental retardation associated with severe developmental delays, distinctive facial features, and skeletal changes. It has an estimated prevalence of 1 in 50000 to 1 in 100000 male infants [1]. The condition was firstly described, independently, by Coffin et al. in 1966 [2] and by Lowry et al. in 1971 [3].

Some of the typical facial features of the disorder include a prominent forehead, hypertelorism, downwardslanting palpebral fissures, epicanthic folds, wide mouth, thick everted lips, broad nose with thick nasal septum and hypodontia. Affected individuals also have broad, soft, and fleshy hands with short tapering fingers. Other reported symptom is a short horizontal crease in the hypothenar region. Skeletal deformities like spinal kyphosis/scoliosis and pectus carinatum or excavatum may present in some patients [4-6]. Here, we report a case of CLS with a disease-causing mutation. To our knowledge, this is the first case report of this syndrome in Iran with a confirmed molecular diagnosis.

\section{Case Report}

This case study reports a 14-year-old Iranian male patient (Figure 1), who was referred to our genetic counseling center for his dysmorphic features and mental retardation. He was the second child of non-consanguineous and healthy parents. His older brother died in early infancy without diagnosis. Parameters at birth were within the normal range. His developmental progress was delayed; he started walking at age 3 and started speaking at age 4 . At the age of 14, his height was $132 \mathrm{~cm}$ and weighted 35 $\mathrm{kg}$. He had mild intellectual disability and seizure. His hearing was normal. Facial features included prominent forehead, widely spaced and downward-slanting eyes, broad and short nose, flat nasal bridge, wide mouth with full lips and hypodontia (Figure $2 \mathrm{~A}-\mathrm{C}$ ).

There was a short horizontal hypothenar crease on the palmar surface of the hands (Figure 2D). The hands were broad with short puffy tapered fingers, which were wide at the base and narrow distally (Figure 2E). His karyotype was normal and pedigree analysis showed that none of the other family members had the same anomalies.

To explore the genetic basis of the disease, we collected peripheral blood samples of the patient and his parents,

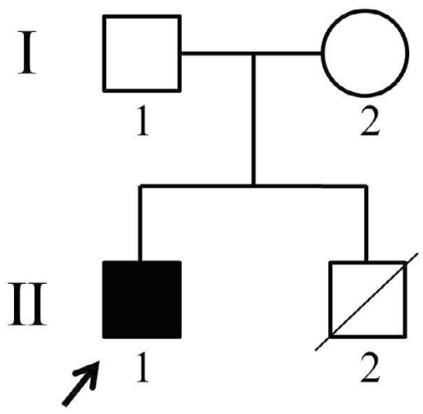

Figure 1. Pedigree of the family Iranian Rehabilitation Journal Symbols are as follows: Circle: Female; Squares: Males; Filled: Affected individual; Empty: Healthy; Slash: Deceased; An arrow indicates the proband. 

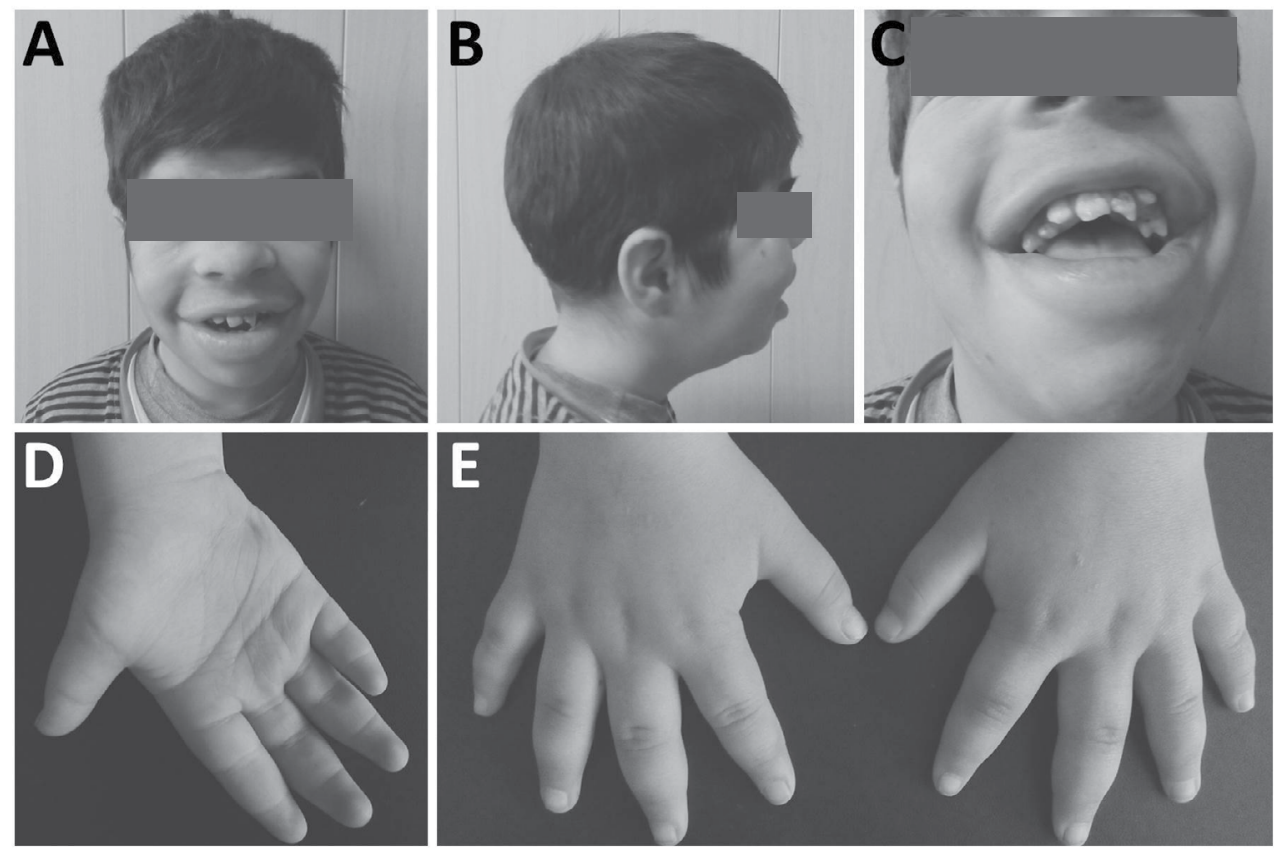

Figure 2. Clinical photographs of the patient

Iranian Rehabilitation Journal

(A-C): Facial views of the patient: Note the prominent forehead, hypertelorism, downward-slanting eyes, broad and short nose, flat nasal bridge, wide mouth, thick lips and hypodontia. (D): Palmar view of the hand showing a hypothenar crease. (E) Dorsal view of the hands showing short, broad and tapering fingers.

after obtaining written informed consent. Genomic DNA was extracted from whole blood by standard methods. By whole exome sequencing, we identified a hemizygous missense variant in exon 22 (c. $2185 \mathrm{C}>\mathrm{T}$; p. Arg729Trp) of RPS6KA3 gene in the patient (Figure 3). The mutation detection was validated by PCR-Sanger sequencing. Segregation analysis showed that both asymptomatic parents do not have this mutation. These findings confirmed the de novo nature of detected mutation in the patient.

\section{Discussion}

We reported a 14-year-old Iranian boy with clinical CLS phenotypes. Genetic testing revealed a hemizygous mutation in the RPS6KA3 gene, leading to the substitution of C-to-T at nucleotide 2185 in exon 22, resulting in an Arg-729 to Trp substitution. To the best of our knowledge, this is the first report of molecularly confirmed case of CLS in the Iranian population.

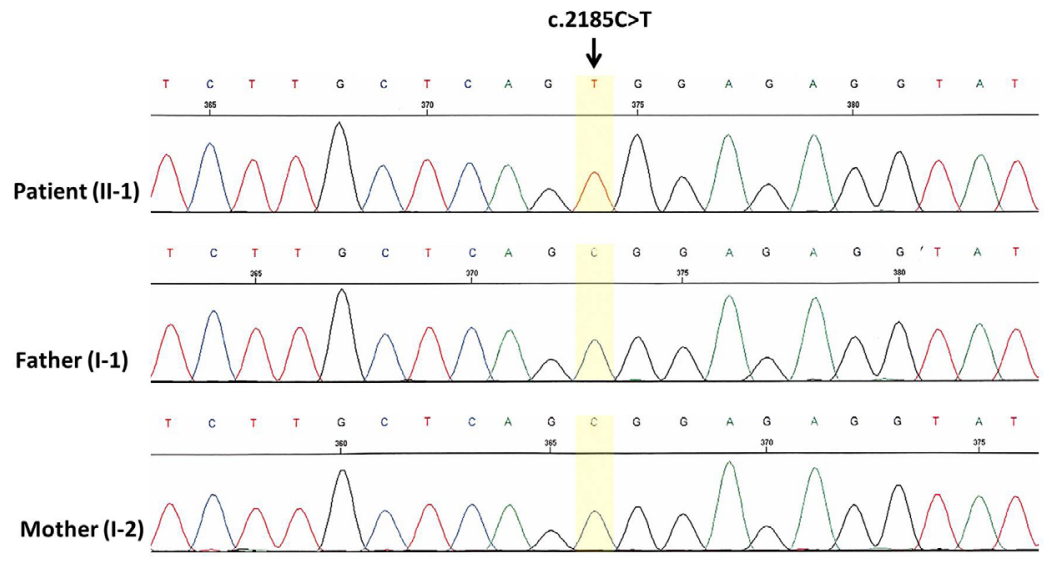

Figure 3. Sanger sequencing chromatogram revealing the pathogenic RPS6KA3 mutation (c.2185C $>\mathrm{T} ;$; p. Argian Rithabilitation『lournal

The parents are normal. 
RPS6KA3 gene (OMIM 300075), which encodes ribosomal S6 kinase-2 (RSK2) is located on the short arm of chromosome X (Xp22.2) and contains 22 exons [7, 8]. RSK2 protein belongs to a highly conserved family of growth factor-activated serine/threonine kinases known as RSK family, which acts at the distal end of the RasMAPK signaling pathway [9]. The RSK family consists of 4 members (RSK1-4) and regulates several important cellular processes, such as cellular proliferation, differentiation, survival, stress response and apoptosis [10].

RSKs are composed of 2 non-identical functional kinase catalytic domains, linked together by a 100-amino acid linker region. Carboxyl (C)-terminal kinase domain is necessary for enzymatic activation of the amino (N)-terminal kinase domain through autophosphorylation. The activated $\mathrm{N}$-terminal domain phosphorylates substrates [11].

Mutations in the RPS6KA3 gene are extremely heterogeneous and have been detected in approximately $50 \%$ of CLS patients. Over 140 distinct inactivating mutations have been reported so far. Identified mutations are distributed over the entire gene and include missense, nonsense, splicing errors, short deletions and insertions, large intragenic deletions, and large duplications [12-15]. About $80 \%$ of the mutations are de novo and sporadic $[1,4]$.

The identified mutation in our patient was previously reported as a pathogenic variant by Delaunoy et al. [12], who described a moderately mentally retarded CLS patient that suffered from deafness and scoliosis, whereas our patient was a mild mentally retarded child, with neither of these symptoms. In contrast, seizure characteristic of the current patient were not found in the previous case. Altogether, further studies are necessary in order to elucidate the phenotypic heterogeneity of CLS with RPS6KA3 mutations.

\section{Conclusion}

In conclusion, we described the clinical and molecular features of a male Iranian child with CLS due to a de novo missense mutation (c.2185C $>$ T; p.Arg729Trp) in exon 22 of RPS6KA3 gene, displaying a mild mental retardation, seizure and typical facial features, also compared the case with a previous report of similar substitution. This comparison showed that some of the symptoms were different in each case. In addition to $R P$ $S 6 K A 3$ mutations, other factors seem to affect the disease severity and may need to be explored in a larger study on patients with CLS.

\section{Ethical Considerations}

\section{Compliance with ethical guidelines}

The authors have completely considered the ethical issues including informed consent, plagiarism, misconduct, data fabrication and/or falsification, double publication and/or submission, and redundancy.

\section{Funding}

This study was financially supported by Welfare Organization of Zanjan, Iran.

\section{Conflict of interest}

The authors declare that there is no conflict of interest regarding the publication of this paper.

\section{Acknowledgements}

The authors appreciate the patient and his family for their assistance and consent for this case report. A special thanks also goes to Golnesa Aghamohammadi for her support.

\section{References}

[1] Pereira PM, Schneider A, Pannetier S, Heron D, Hanauer A Coffin-Lowry syndrome. European Journal of Human Genetics. 2010; 18(6):627-33. [DOI:10.1038/ejhg.2009.189] [PMID] [PMCID]

[2] Coffin GS, Siris E, Wegienka LC. Mental retardation with osteocartilaginous anomalies. American Journal of Diseases of Children. 1966; 112(3):205-13. [DOI:10.1001/archpedi.1966.02090120073006]

[3] Lowry B, Miller JR, Fraser FC. A new dominant gene mental retardation syndrome: Association with small stature, tapering fingers, characteristic facies, and possible hydrocephalus. American Journal of Diseases of Children. 1971; 121(6):496-500. [DOI:10.1001/archpedi.1971.02100170078009] [PMID]

[4] Hanauer A, Young ID. Coffin-Lowry syndrome: Clinical and molecular features. Journal of Medical Genetics. 2002; 39(10):70513. [DOI:10.1136/jmg.39.10.705] [PMID] [PMCID]

[5] Herrera-Soto JA, Santiago-Cornier A, Segal LS, Ramirez N, Tamai J. The musculoskeletal manifestations of the Coffin-Lowry syndrome. Journal of Pediatric Orthopaedics. 2007; 27(1):85-9. [DOI:10.1097/01.bpo.0000187994.94515.9d]

[6] Welborn M, Farrell S, Knott P, Mayekar E, Mardjetko S. The natural history of spinal deformity in patients with Coffin-Lowry syndrome. Journal of Children's Orthopaedics. 2018; 12(1):70-5. [DOI:10.1302/1863-2548.12.170101] [PMID] [PMCID]

[7] Hanauer A, Alembik Y, Gilgenkrantz S, Mujica P, NivelonChevallier A, Pembrey ME, et al. Probable localisation of the Coffin-Lowry locus in Xp22. 2-P22. 1 by multipoint linkage analy- 
sis. American Journal of Medical Genetics. 1988; 30(1-2):523-30. [DOI:10.1002/ajmg.1320300154] [PMID]

[8] Biancalana V, Briard ML, David A, Gilgenkrantz S, Kaplan $\mathrm{J}$, Mathieu M, et al. Confirmation and refinement of the genetic localization of the Coffin-Lowry syndrome locus in Xp22. 1-p22. 2. American Journal of Human Genetics. 1992; 50(5):981-7. [PMID] [PMCID]

[9] Hauge C, Frödin M. RSK and MSK in MAP kinase signalling. Journal of Cell Science. 2006; 119(15):3021-3. [DOI:10.1242/ jcs.02950] [PMID]

[10] Zeniou M, Ding T, Trivier E, Hanauer A. Expression analysis of RSK gene family members: The RSK2 gene, mutated in Coffin-Lowry syndrome, is prominently expressed in brain structures essential for cognitive function and learning. $\mathrm{Hu}-$ man Molecular Genetics. 2002; 11(23):2929-40. [DOI:10.1093/ hmg/11.23.2929] [PMID]

[11] Frödin M, Gammeltoft S. Role and regulation of 90 kDa Ribosomal S6 Kinase (RSK) in signal transduction. Molecular and Cellular Endocrinology. 1999; 151(1-2):65-77. [DOI:10.1016/ S0303-7207(99)00061-1]

[12] Delaunoy J, Dubos A, Marques Pereira P, Hanauer A. Identification of novel mutations in the RSK2 gene (RPS6KA3) in patients with Coffin-Lowry syndrome. Clinical Genetics. 2006; 70(2):161-6. [DOI:10.1111/j.1399-0004.2006.00660.x] [PMID]

[13] Pereira PM, Heron D, Hanauer A. The first large duplication of the RSK2 gene identified in a Coffin-Lowry syndrome patient. Human Genetics. 2007; 122(5):541-3. [DOI:10.1007/s00439-0070424-1] [PMID]

[14] Madrigal I, Rodríguez-Revenga L, Armengol L, González E Rodriguez B, Badenas C, et al. X-chromosome tiling path array detection of copy number variants in patients with chromosome X-linked mental retardation. BMC Genomics. 2007; 8:443. [DOI:10.1186/1471-2164-8-443] [PMID] [PMCID]

[15] Jurkiewicz D, Jezela-Stanek A, Ciara E, Piekutowska-Abramczuk D, Kugaudo M, Gajdulewicz M, et al. Four novel RSK2 mutations in females with Coffin-Lowry syndrome. European Journal of Medical Genetics. 2010; 53(5):268-73. [DOI:10.1016/j. ejmg.2010.07.006] [PMID] 